\title{
Abnormal expression of microRNA-575 leads to missed abortion through regulating apoptosis and angiogenesis
}

\author{
SHUQIN XIA ${ }^{1-3}$, YIHUI ZHEN ${ }^{3}$, HONGSHENG MA $^{3}$ and AIMING WANG ${ }^{1,2}$ \\ ${ }^{1}$ Department of Gynecology and Obstetrics, Southern Medical University, Guangzhou, Guangdong 510515; \\ ${ }^{2}$ Department of Gynecology and Obstetrics, Inner Mongolia People's Hospital, Hohhot, Inner Mongolia 010017; \\ ${ }^{3}$ Department of Gynecology and Obstetrics, Navy General Hospital PLA China, Beijing 100048, P.R. China
}

Received July 17, 2016; Accepted March 31, 2017

DOI: $10.3892 / \mathrm{etm} .2017 .5086$

\begin{abstract}
Numerous microRNA (miR) are important for placental development and function. miR-575 has been demonstrated to be upregulated in maternal placenta in patients who have experienced a miscarriage. The present study aimed to explore the role of abnormal expression of miR-575 in missed abortion (MA) and to further analyze the potential molecular mechanisms. Embryo villus tissue samples were extracted from 10 childless women with MA and 10 fertile women without a history of MA. Additionally, human choriocarcinoma cells, JEG-3, were used in the present study, which were transfected with miR-575 mimic, inhibitor and scramble. The expression of miR-575 in embryo villus tissues and in JEG-3 cells was detected by reverse transcription-quantitative polymerase chain reaction (RT-qPCR). Apoptosis in villus tissues of patients with MA and in JEG-3 cells of miR-575 mimic, inhibitor and scramble groups were detected by flow cytometry. Furthermore, the expression levels of apoptosis-related proteins, including B-cell lymphoma 2 (Bcl-2), Bcl-2-associated X protein (Bax) and phosphorylated (p)-p53, and angiogenesis-related proteins, including vascular endothelial growth factor (VEGF) and angiopoietin 2 (Ang-2), were measured by RT-qPCR and western blotting. Additionally, the target of miR-575 was predicted and clarified by luciferase reporter assay. miR-575 was significantly overexpressed in MA villus tissue compared with normal tissue $(\mathrm{P}<0.05)$. The percentage of apoptotic cells in MA embryo villus tissue was significantly higher than that in normal tissue $(\mathrm{P}<0.05)$. After JEG-3 cells were transfected with miR-575 inhibitor, the expression of miR-575 and the percentage of apoptotic cells decreased significantly compared with the control $(\mathrm{P}<0.05)$. MiR-575 suppression significantly increased the expression of
\end{abstract}

Correspondence to: Professor Aiming Wang, Department of Gynecology and Obstetrics, Southern Medical University, 1023 South Shatai Road, Guangzhou, Guangdong 510515, P.R. China

E-mail: aimingwang33@126.com

Key words: missed abortion, microRNA-575, cell apoptosis, angiogenesis
Bcl-2 $(\mathrm{P}<0.05)$, and decreased the expressions of $\operatorname{Bax}(\mathrm{P}<0.05)$ and p-p53 $(\mathrm{P}<0.01)$ compared with the control. Furthermore, miR-575 suppression significantly increased the expressions of angiogenesis-related proteins, Ang-2 and VEGF $(\mathrm{P}<0.01)$. Superoxide dismutase 2 was identified as the target of miR-575. Therefore, abnormal expression of miR-575 may lead to MA through regulating apoptosis and angiogenesis. Inhibition of miR-575 may inhibit apoptosis and promote angiogenesis in MA.

\section{Introduction}

Missed abortion (MA) is a common obstetrical and gynecological disease and is a complication of early pregnancy, which is characterized by the arrest of embryonic or fetal development (1). The prevalence of MA is $2 \%$ in singleton pregnancies and $\sim 90 \%$ of MA cases occur in the first trimester (2). In China, MA is a widespread and serious clinical occurrence that not only damages the woman's health, but also has a great influence on population quality (3). Currently, the etiology of MA is not fully understood. An increasing number of researchers are focusing on the underlying pathogenesis of this disorder.

Several causes, such as chromosomal anomalies, uterine abnormalities, hormonal problems and autoimmune disorders, have been demonstrated to be associated with the occurrence of MA (4); however, no one cause is identified in certain cases. It has been reported that normal pregnancy has a certain degree of trophoblast apoptosis, which is conducive to the formation and development of villi and chorionic villi branch (5). However, excessive apoptosis of trophoblasts may result in villi dysplasia or degeneration of cytotrophoblast cells, or even pregnancy failure and MA (6-8). Additionally, a study by Chen et al (9) reported that sufficient angiogenesis in villi has an important role in the maintenance of early pregnancy. Therefore, apoptosis and angiogenesis may be associated with the occurrence of MA.

A study by Nelissen et al (10) revealed that epigenetic regulation governing the control of gene expression is an important factor of placental development and function. Genome-wide analysis has demonstrated that there are $>600$ microRNA (miR) expressed in human placenta (11). Notably, a study by Hosseini (12) investigated the differentially expressed $\mathrm{miR}$ in maternal plasma and placenta in patients that had experienced 
a miscarriage and observed that the expression level of miR-575 was upregulated in these patients. However, the role of miR-575 in MA has not been investigated.

The present study investigated the relative expression level of miR-575 in embryo villus tissues in patients with MA. In addition, the effects of miR-575 on the apoptosis and angiogenesis of villus cells were assessed. The present study aimed to explore the role of miR-575 in MA and to further analyze the potential molecular mechanisms.

\section{Materials and methods}

Patients. A total of 10 childless women aged 25-30 years with MA at $\sim 6$ gestational weeks at Southern Medical University (Guangzhou, China) between June 2013 and December 2015 were included in the present study. An additional 10 fertile women aged between 25-30 years with $\geq 1$ child and no history of MA were used as controls. Embryo villus tissue samples of the 20 cases were extracted, as previously described (13). Samples were snap frozen in liquid nitrogen and stored at $-80^{\circ} \mathrm{C}$ until use. The present study was approved by the local Ethics Committee at the hospital of Southern Medical University and all patients provided written informed consent.

Cell line and cell transfection. Human choriocarcinoma cell line, JEG-3, was used in the present study, which was purchased from American Type Culture Collection (Manassas, VA, USA). The cell line was cultured in Dulbecco's modified Eagle medium (DMEM)/F12 (Gibco; Thermo Fisher Scientific, Inc., Waltham, MA, USA) supplemented with $10 \%$ fetal bovine serum (Gibco; Thermo Fisher Scientific, Inc.) and $100 \mathrm{U} / \mathrm{ml}$ penicillin (Sigma-Aldrich; Merck KGaA, Darmstadt, Germany) in an atmosphere of $5 \% \mathrm{CO}_{2}$ at $37^{\circ} \mathrm{C}$.

For cell transfection, miR-575 mimic, inhibitor and scramble, and the pc-SOD2 (pcDNA 3.1 vector with SOD2 coding sequence) were purchased from Sangon Biotech Co., Ltd., (Shanghai, China). Vectors were transfected into JEG-3 cells using Lipofectamine 2000 (Invitrogen; Thermo Fisher Scientific, Inc.), according to the manufacturer's instructions.

Reverse transcription-quantitative polymerase chain reaction (RT-qPCR). Total RNA from tissues (MA tissues and control tissues) or the cultured cells (JEG-3 cells transfected with or without vectors) was isolated using TRIzol reagent (Invitrogen; Thermo Fisher Scientific, Inc.), according to the manufacturer's protocol. The concentration and purity of the isolated DNA was determined using an SMA 400 UV-VIS spectrophotometer (Merinton, Ltd., Shanghai, China). Purified RNA $(0.5 \mu \mathrm{g} / \mu \mathrm{l})$ was mixed with nuclease-free water for the cDNA synthesis using Script cDNA Synthesis kit (Bio-Rad Laboratories, Inc., Hercules, CA, USA). Targets (mRNA and miR) were synthesized using the SYBR ExScriptqRT-PCR kit (Takara Biotechnology Co., Ltd., Dalian, China). Primers used for target amplification are demonstrated in Table I. U6 and GAPDH were used as internal controls. The reaction system was in a final volume of $20 \mu \mathrm{l}$, which contained the following: $1 \mu \mathrm{l}$ cDNA, $10 \mu \mathrm{l}$ SYBR Premix EX Taq, $1 \mu \mathrm{l}$ forward primer $(10 \mu \mathrm{M}), 1 \mu \mathrm{l}$ reverse primer $(10 \mu \mathrm{M})$ and $7 \mu \mathrm{l} \mathrm{ddH}_{2} \mathrm{O}$. The amplification system was as follows: Denatureation at $95^{\circ} \mathrm{C}$ for $5 \mathrm{~min}$, followed by 40 cycles at $95^{\circ} \mathrm{C}$ for $30 \mathrm{sec}$, annealing at

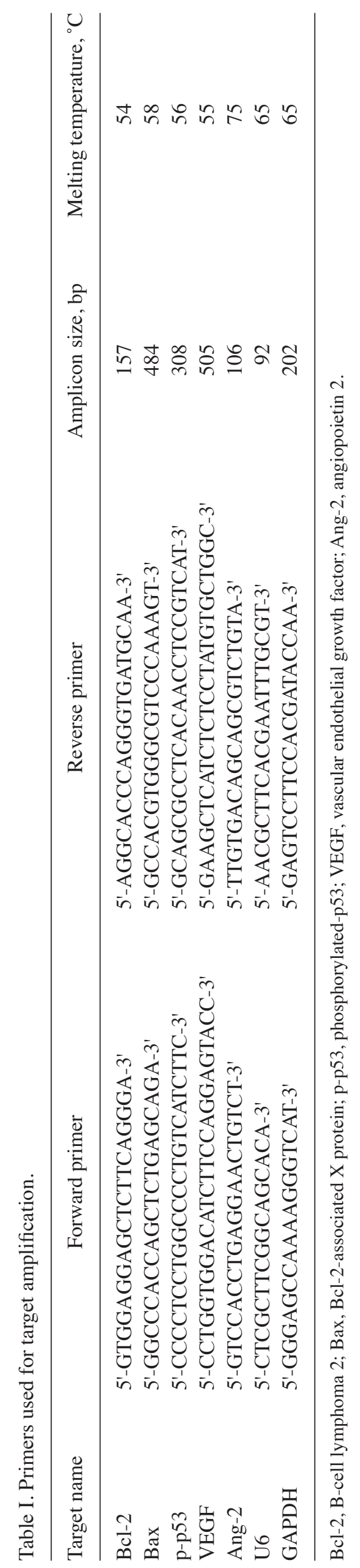


A

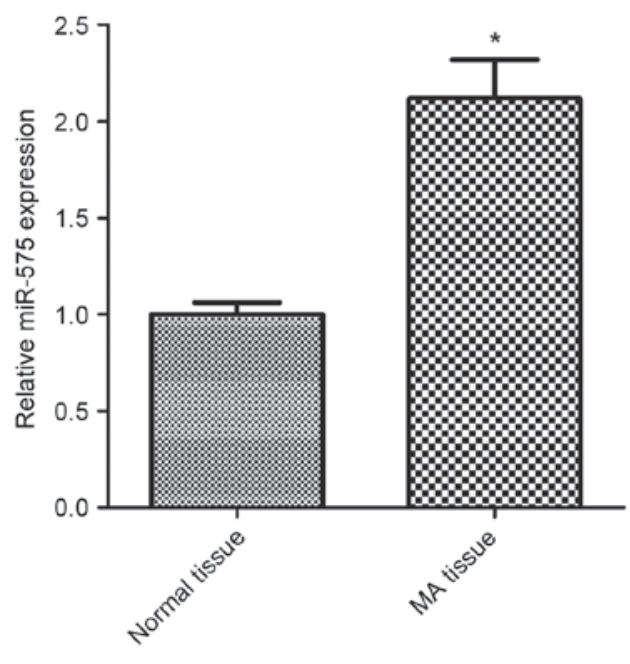

B

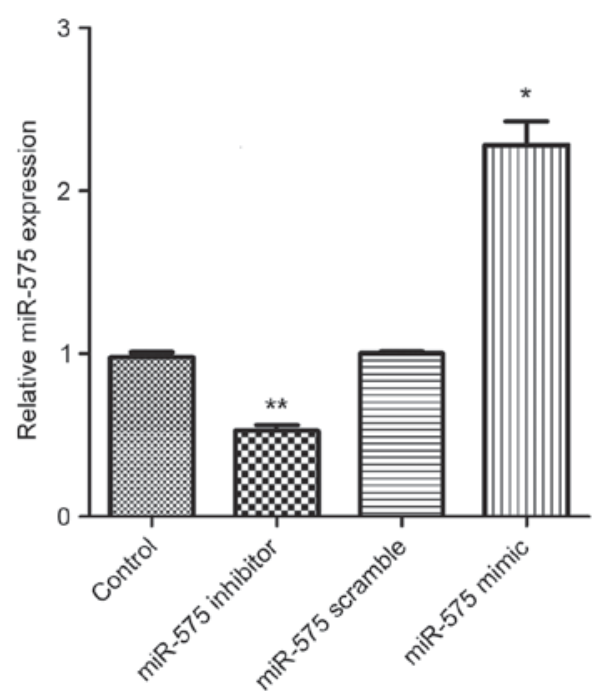

Figure 1. Expression level of miR-575 in (A) placental villus tissue and (B) transfected JEG-3 cells determined by reverse transcription-quantitative polymerase chain reaction. U6 served as the internal control. Experiments were repeated three times. Data are expressed as the mean + standard deviation. (A) " $\mathrm{P}<0.05 \mathrm{vs}$. normal tissue; (B) $\mathrm{P}<0.05$ and ${ }^{* *} \mathrm{P}<0.01$ vs. control. miR, microRNA; MA, missed abortion.

$54^{\circ} \mathrm{C}$ for $30 \mathrm{sec}$ and extension at $72^{\circ} \mathrm{C}$ for $1 \mathrm{~min}$ and a final extension at $72^{\circ} \mathrm{C}$ for $10 \mathrm{~min}$. The $\mathrm{mRNA} / \mathrm{miR}$ expression levels were detected by SYBR Green-based qPCR (SYBR Green Master mix; Thermo Fisher Scientific, Inc.). Three repeats were performed. The $2^{-\Delta \Delta \mathrm{Cq}}$ method (14) was used to calculate the relative expression levels of mRNA and miR.

Cell apoptosis assay. Cell apoptosis was assayed by flow cytometry using an annexin V-fluorescein isothiocyanate (FITC) cell apoptosis kit (Invitrogen; Thermo Fisher Scientific, Inc.), according to the manufacturer's protocol. Briefly, after transfection for $48 \mathrm{~h}$, cells were cultured with fresh serum-free DMEM/F12 medium at $37^{\circ} \mathrm{C}$ for $12 \mathrm{~h}$. Subsequently, cells were harvested and washed three times ( $5 \mathrm{~min} /$ wash) with phosphate-buffered saline ( $\mathrm{pH} 7.4)$, and then resuspended in staining buffer. Following this, cells were mixed with $5 \mu \mathrm{l}$ annexin-V-FITC and $5 \mu \mathrm{l}$ propidium iodide (PI). After $10 \mathrm{~min}$, the mixtures were analyzed using a FACScan flow cytometer (BD Biosciences, San Jose, CA, USA) and in-built software. Annexin V-positive and PI-negative cells were regarded as apoptotic cells.

Western blot analysis. Cells were lysed with radioimmunoprecipitation assay buffer (Sangon Biotech Co., Ltd.). Subsequently, $50 \mu \mathrm{g}$ protein sample was separated by $10 \%$ SDS-PAGE and blotted onto polyvinylidene difluoride membranes. Following incubation with primary antibodies specific for angiopoietin 2 (Ang-2; ab99971), vascular endothelial growth factor (VEGF; ab32152), B-cell lymphoma 2 (Bcl-2; ab32124), Bcl-2-associated X protein (Bax; ab32503), phosphorylated-p53 (p-p53; ab1101) and superoxide dismutase 2 (SOD2; ab13534) (all 1:1,000 dilution) at $4^{\circ} \mathrm{C}$ overnight, the membranes were incubated with the appropriate horseradish peroxidase-conjugated secondary antibodies (1:1,000 dilution, catalog no. ab6721) at room temperature for 0.5-1 h. All antibodies were purchased from Abcam (Cambridge, MA, USA). The immunoreactive protein bands were visualized by enhanced chemiluminescence (Amersham; GE Healthcare, Chicago, IL, USA). GAPDH served as the internal control.

Target gene prediction. Bioinformatic analysis was performed to predict the target gene of miR-575, using TargetScanHuman v7.1 software (www.targetscan.org/vert_71).

Luciferase reporter analysis. Vectors of SOD2-3'-untranslated region (UTR), miR-575 inhibitor and scramble were synthesis by Sangon Biotech Co., Ltd. The dual-luciferase reporter plasmids, SOD2-WT (containing the wild-type SOD2 putative 3'-UTR-binding site) and SOD2-Mut (containing the mutant SOD2 3'-UTR) were constructed. Lipofectamine 2000 (Invitrogen; Thermo Fisher Scientific, Inc.) was used to transfect plasmids into cells. Luciferase activities were measured using the dual-luciferase reporter assay system (Promega Corp., Madison, WI, USA) after 48 h of cell transfection. The relative reporter activity was normalized to Renilla luciferase activity.

Statistical analysis. In the present study, all experiments were conducted three times, independently. The data were expressed as the mean \pm standard deviation. Statistical analysis was performed using SPSS v. 19.0 software (IBM Corp., Armonk, NY, USA). All collected data were tested for the normal distribution using one-sample Kolmogorov-Smirnov test. Differences between groups were evaluated using one-way analysis of variance with Tukey's post hoc test. $\mathrm{P}<0.05$ was considered to indicate a statistically significant difference.

\section{Results}

Expression of miR-575 in villus tissue and cells. To investigate the miR-575 expression in villus tissue and cells, RT-qPCR analysis was performed. Results demonstrated that the expression of miR-575 was significantly upregulated in MA placental villus tissues compared with normal tissues $(\mathrm{P}<0.05$; Fig. 1A). 
A

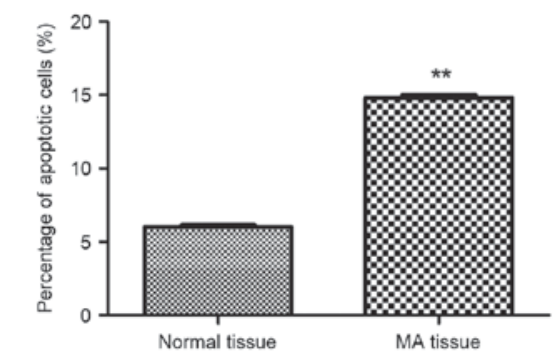

B

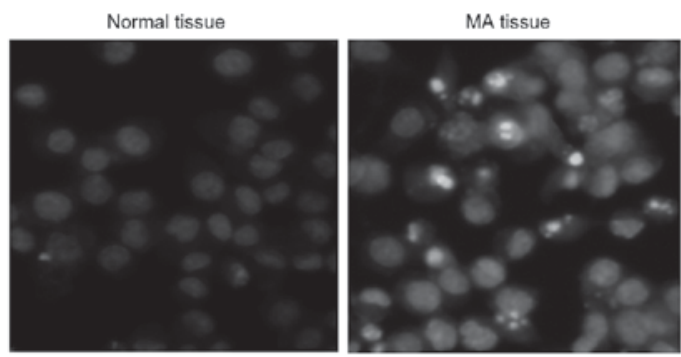

C
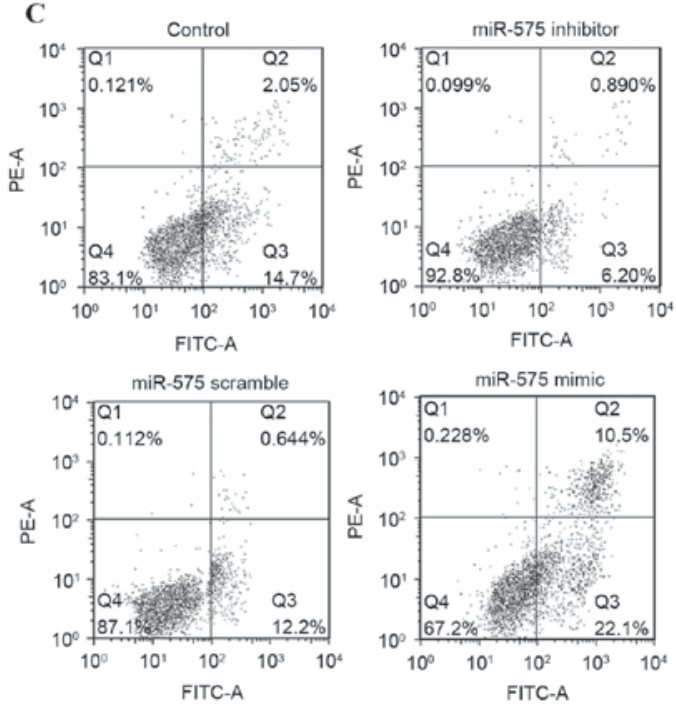

D

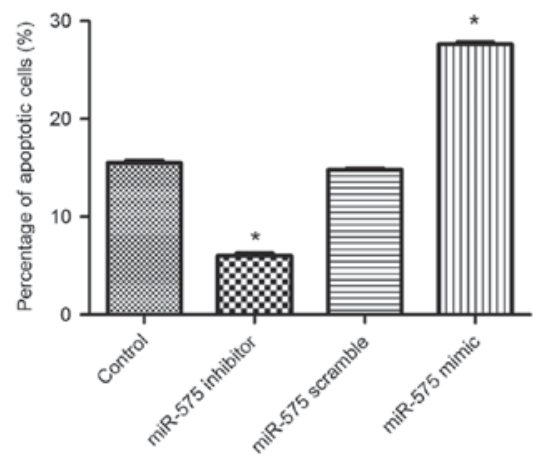

Figure 2. (A) Percentage of apoptotic cells in placental villus tissue assayed by flow cytometry. (B) Fluorescent staining results for apoptotic cells obtained from flow cytometry at magnification, x400. Apoptotic cells were analyzed with Annexin V-FITC. (C and D) The percentage of apoptotic JEG-3 cells following miR-575 inhibition or overexpression assayed by flow cytometry. Experiments were repeated three times. Data are expressed as the mean + standard deviation. ${ }^{* *} \mathrm{P}<0.05$ vs. normal tissue; ${ }^{*} \mathrm{P}<0.05$ vs. control. PE-A, pseudomonas exotoxin A; FITC, fluorescein isothiocyanate; miR, microRNA; MA, missed abortion.

Furthermore, after JEG-3 cells were transfected with miR-575 inhibitor, the expression of miR-575 decreased significantly compared with the control and miR-575 scramble groups $(\mathrm{P}<0.05)$. No significance difference in miR-575 expression was observed between the control and miR-575 scramble transfected groups. Contrastingly, after the JEG-3 cells were transfected with miR-575 mimic, the expression of miR-575 significantly increased compared with the control group ( $\mathrm{P}<0.01$; Fig. 1B).

Effect of miR-575 on cell apoptosis. Excessive cell apoptosis may result in pregnancy failure and MA (8). To characterize the biological significance of miR-575 in MA development, cell apoptosis assays were performed using flow cytometry. As demonstrated in Fig. 2A and B, the percentage of apoptotic cells in MA villus tissue was significantly higher than that in normal tissue $(\mathrm{P}<0.05)$. Transfection experiments also indicated that overexpression of miR-575 significantly promoted the apoptosis of JEG-3 cells compared with the control group $(\mathrm{P}<0.01$; Fig. $2 \mathrm{C}$ and $\mathrm{D})$. No significant difference in cell apoptosis was observed between the control and miR-575 scramble transfected groups. When JEG-3 cells were transfected with miR-575 inhibitor, the percentage of apoptotic cells decreased significantly compared with the other three groups $(\mathrm{P}<0.05)$.

Effect of miR-575 on cell apoptosis-related protein expression. To further explore the underlying molecular mechanisms of miR-575 on cell apoptosis, the expression levels of apoptosis-related proteins, Bcl-2, Bax and p-p53, were measured using RT-qPCR and western blot analyses. Results demonstrated that overexpression of miR-575 significantly decreased the mRNA expression level $(\mathrm{P}<0.05)$ and the protein expression level of Bcl-2 compared with that in the control group. Also, overexpression of miR-575 significantly increased the expression levels of Bax and p-p53 compared with that in the control group $(\mathrm{P}<0.05$ and $\mathrm{P}<0.01$, respectively). No significant difference in expression was observed between the control and miR-575 scramble transfected groups. However, miR-575 suppression significantly increased the expression level of Bcl-2 $(\mathrm{P}<0.05)$, and decreased the expression levels of Bax and p-p53 compared with the other three groups $(\mathrm{P}<0.05$ and $\mathrm{P}<0.01$, respectively; Fig. 3A and B).

Effect of miR-575 on angiogenesis-related protein expression. It has been reported that sufficient angiogenesis has an important role in the maintenance of early pregnancy (9). Therefore, the role of miR-575 in angiogenesis was investigated by detecting the expression levels of angiogenesis-related proteins, Ang-2 and VEGF, using RT-qPCR and western blot analyses. As demonstrated in Fig. 4A and B, miR-575 overexpression significantly decreased the mRNA expression levels $(\mathrm{P}<0.05)$ and markedly decreased the protein expression levels of both Ang-2 and VEGF compared with the control group. However, after JEG-3 cells were transfected with miR-575 inhibitor, the expression levels of both Ang-2 and VEGF increased 
A

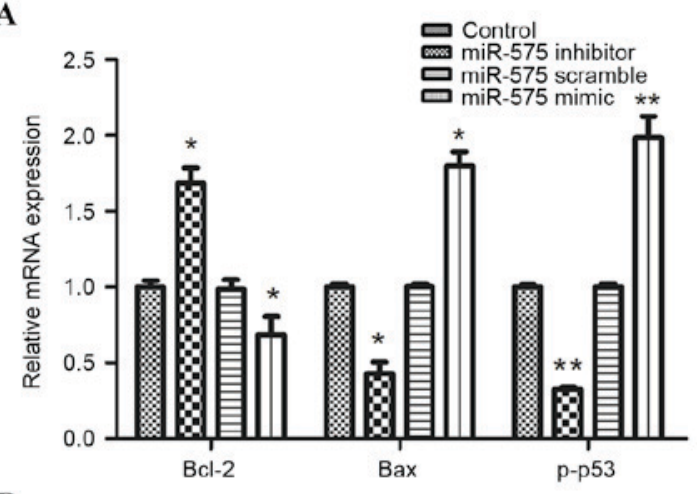

B

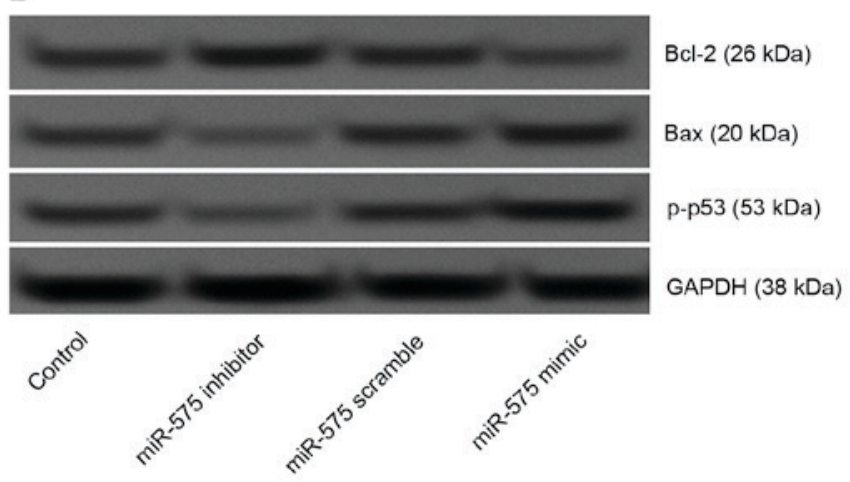

Figure 3. Expression levels of apoptosis-related proteins, Bcl-2, Bax and p-p53, following miR-575 inhibition or overexpression assayed by (A) reverse transcription-quantitative polymerase chain reaction and (B) western blot analysis. GAPDH served as the internal control. Experiments were repeated three times. Data are expressed as the mean + standard deviation. " $\mathrm{P}<0.05$ and ${ }^{* *} \mathrm{P}<0.01$ vs. control. Bcl-2, B-cell lymphoma 2; Bax, Bcl-2-associated X protein; p-p53, phosphorylated-p53; miR, microRNA.

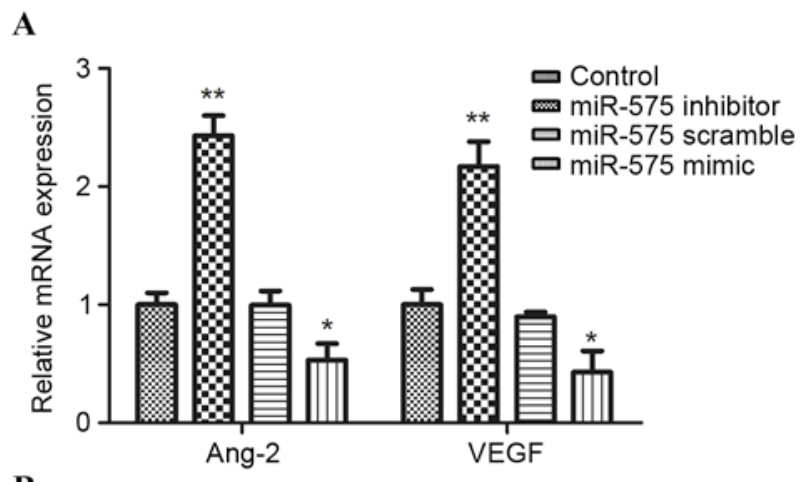

B

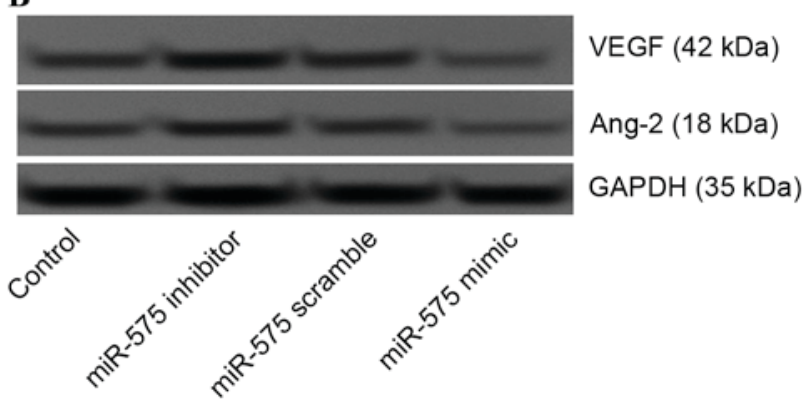

Figure 4. Expression levels of angiogenesis-related proteins, VEGF and Ang-2, following miR-575 inhibition or overexpression assayed by (A) reverse transcription-quantitative polymerase chain reaction and (B) western blot analysis. GAPDH served as the internal control. Experiments were repeated three times. Data are expressed as the mean + standard deviation. ${ }^{*} \mathrm{P}<0.05$ vs. control; ${ }^{* *} \mathrm{P}<0.01$ vs. miR-575 mimic group. VEGF, vascular endothelial growth factor; Ang-2, angiopoietin 2; miR, microRNA.

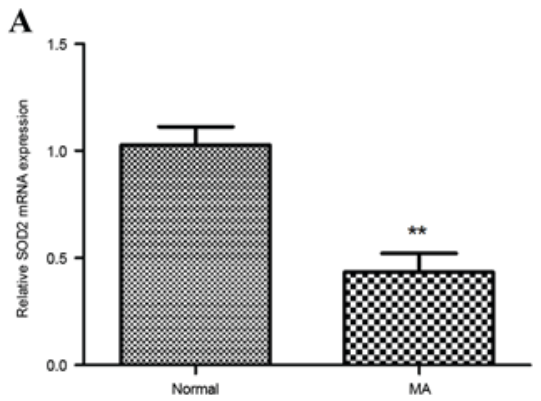

B

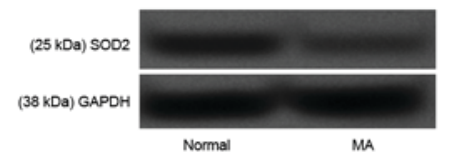

Figure 5. The expression of SOD2 in placental villus tissue assayed by (A) reverse transcription-quantitative polymerase chain reaction and (B) western blot analysis. GAPDH served as the internal control. Experiments were repeated three times. Data are expressed as the mean + standard deviation. ${ }^{* *} \mathrm{P}<0.01$ vs. normal tissue. SOD2, superoxide dismutase 2; MA, missed abortion.

significantly compared with the miR-575 scramble and mimic groups $(\mathrm{P}<0.01)$.

SOD2 is a direct target for miR-575 and miR-575 regulates $M A$ by targeting SOD2. Research has demonstrated that SOD is downregulated in trophoblasts from women with MA (15). In the present study, the expression of SOD2 in villus tissue of MA patients was investigated by RT-qPCR and western blot analyses. As demonstrated in Fig. 5A and B, SOD2 was significantly downregulated in villus tissue from patients with MA compared with normal tissues $(\mathrm{P}<0.01)$.

According to TargetScanHuman software (targetscan. org), SOD2 was predicted to be the target gene of miR-575 (Fig. 6A). Luciferase reporter analysis demonstrated that the relative luciferase activity of the reporter that contained SOD2 wild type 3'-UTR reduced significantly in miR-575-inhibitortransfected cells compared with the miR-control transfected cells containing the SOD2 wild type 3 'UTR $(\mathrm{P}<0.01$; Fig. $6 \mathrm{~B})$. Additionally, the relative expression of SOD2 decreased significantly when miR-575 was overexpressed and increased significantly when miR-575 was silenced compared with the control group $(\mathrm{P}<0.01$; Fig. $6 \mathrm{C})$. These results indicated that miR-575 may directly regulate the SOD2 gene. Furthermore, after pc-SOD2 was transfected into cells, it was demonstrated that SOD2 expression was significantly increased compared with the control group ( $\mathrm{P}<0.01$; Fig. 7A). Notably, further investigation indicated that overexpressed SOD2 was able to significantly reverse the inhibiting effect of miR-575 inhibitor on apoptosis $(\mathrm{P}<0.01$; Fig. 7B) and expression of angiogenesis factors, Ang-2 and VEGF (Fig. 7C). These results suggested that miR-575 may regulate apoptosis and angiogenesis by targeting the SOD2 gene.

\section{Discussion}

The present study demonstrated that miR-575 was significantly overexpressed in MA embryo villus tissue compared 
A

\section{B}

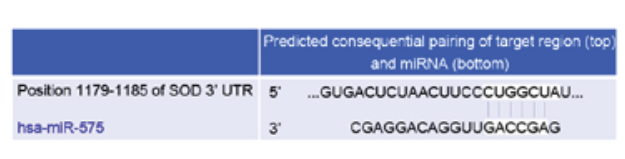

C

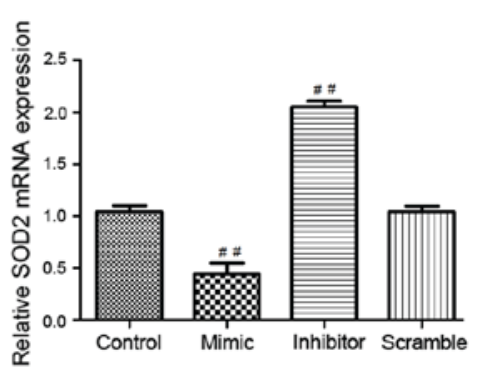

$(25 \mathrm{kDa})$ SOD2

(38 kDa) GAPDH

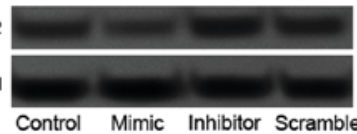

Figure 6. (A) Gene sequences of SOD2 regulated by miR-575. (B) Relative luciferase activities in WT 3'-UTR of SOD2 and mut SOD2 3'-UTR in transfected cells. (C) Relative expression levels of SOD2 in transfected cells assayed by reverse transcription-quantitative polymerase chain reaction and western blot analysis. Data are expressed as the mean + standard deviation. ${ }^{* *} \mathrm{P}<0.01$ vs. miR-control transfected cells containing the SOD2 wild type $3^{\prime}-\mathrm{UTR}$; ${ }^{\# *} \mathrm{P}<0.01$ vs. control. SOD2, superoxide dismutase 2; WT, wild type; mut, mutant; UTR, untranslated region; miR, microRNA.

A

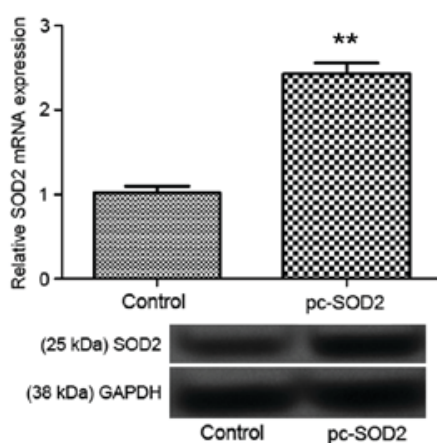

B
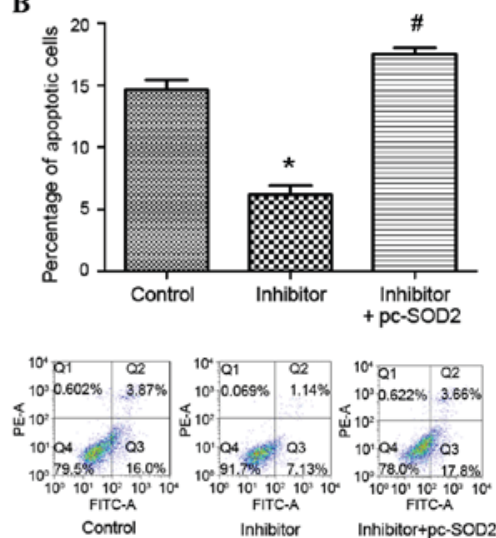

C

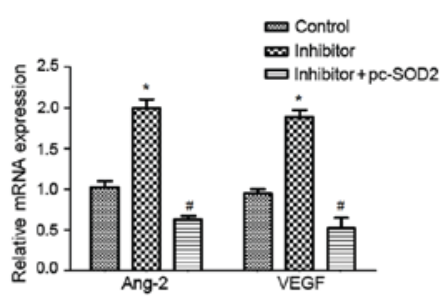

(18 kDa) Ang-2

(42 kDa) VEGF

(38 kDa) GAPDH

Control Inhibitor Inhibitor

Figure 7. (A) Expression of SOD2 after cells were transfected with pc-SOD2 assayed by RT-qPCR and western blot analysis. (B) Percentage of apoptotic JEG-3 cells following transfection with pc-SOD2, assayed by flow cytometry. (C) Expression levels of angiogenesis-related proteins, VEGF and Ang-2, after cells were transfected with pc-SOD2, assayed by RT-qPCR and western blot analysis. Experiments were repeated three times. Data are expressed as the mean + standard deviation. ${ }^{*} \mathrm{P}<0.05$ and ${ }^{* *} \mathrm{P}<0.01$ vs. control; ${ }^{*} \mathrm{P}<0.05$ vs. inhibitor group. SOD2, superoxide dismutase 2; pc-SOD2, pcDNA 3.1 vector with SOD2 coding sequence; RT-qPCR, reverse transcription-quantitative polymerase chain reaction; VEGF, vascular endothelial growth factor; Ang-2, angiopoietin 2; FITC, fluorescein isothiocyanate; PE-A, pseudomonas exotoxin A. 
with normal tissues. The percentage of apoptotic cells in MA embryo villus tissues was significantly higher than that in normal tissues. Transfection experiments revealed that miR-575 overexpression was able to promote the apoptosis of JEG-3 cells and decreased the expression of angiogenesis-related proteins. After JEG-3 cells were transfected with miR-575 inhibitor, the percentage of apoptotic cells decreased significantly, and the expression of angiogenesis-related proteins, Ang-2 and VEGF, significantly increased. To the best of our knowledge, the present study is the first to investigate the role of miR-575 in MA.

Various studies have suggested that the level of apoptosis is critically important for the successful development of normal pregnancy (7-17). A low level of apoptosis in placental villi tissues is a normal physiological phenomenon (8); however, a high level of apoptosis may result in miscarriage $(18,19)$. In the present study, miR-575 was overexpressed in MA embryo villus tissue, suggesting the possible role of miR-575 in promoting the development of MA. Notably, miR-575 overexpression promoted the apoptosis of JEG-3 cells, suggesting that miR-575 overexpression may have an important role in the cell apoptosis in MA.

Previous research has reported that a series of genes coordinately regulate apoptosis and proliferation during pregnancy (20). For example, p53, a key regulator of apoptosis and the cell cycle, has been demonstrated to be overexpressed in the chorionic villi of females with spontaneous abortions or MA $(21,22)$. In the present study, p-p53 was upregulated in the miR-575 mimic group. However, when JEG-3 cells were transfected with miR-575 inhibitor, the expression of p-p53 decreased significantly, indicating that miR-575 may have an important role in MA by regulating the apoptosis of villi cells.

In addition to $\mathrm{p} 53$, Bax, one of the pro-apoptotic members of the Bcl-2 family demonstrated reduced expression in the miR-575 inhibitor group. Contrastingly, the expression of anti-apoptotic Bcl-2 increased significantly following miR-575 inhibition. Notably, previous research has revealed that the abnormal expression of Bcl-2 and Bax in placental villi tissues is associated with recurrent abortion (23). These results further indicated the role miR-575 in the apoptosis of villi cells.

A successful pregnancy not only depends on apoptosis, but also depends on sufficient villous angiogenesis, which is able to supply adequate oxygen and nutrients $(24,25)$. Abnormal angiogenesis is considered as an important contributor to MA (26). VEGF is believed to be a powerful angiogenesis promoter, which has a critical role in the development and maintenance of the vasculature (27). A study by Çöl-Madendag et al (28) revealed that decreased expression of VEGF in placental villi and decidua may be associated with early abortion. Additionally, the angiopoietin family is another important angiogenic protein family, which has been demonstrated to be critically involved in angiogenesis, particularly in the female reproductive tract (29-31). Ang-2, a member of the angiopoietin family, may be expressed in the early placenta in normal and pathological pregnancy $(32,33)$. High levels of Ang-2 may confer protection to the placenta (34). The present study demonstrated that overexpression of miR-575 significantly decreased the expression of VEGF and Ang-2. After miR-575 was inhibited, the expression levels of VEGF and Ang-2 increased again, indicating the regulatory role of miR-575 in angiogenesis in MA.

It has been reported that oxidative stress is associated with MA (35). SOD is an important antioxidant that catalyzes the dismutation of superoxide into hydrogen peroxide and oxygen molecules, thereby scavenging free radicals and preventing oxygen toxicity (36). Notably, alterations to SOD concentration have been closely related to spontaneous abortion (36). A study by Zhu et al (15) indicated that SOD was downregulated in trophoblasts from women with MA. In the present study, SOD2 was suggested to be a target gene of miR-575, and was downregulated in MA tissue. Further investigation demonstrated that overexpression of SOD2 was able to reverse the inhibiting effects of miR-575 inhibitor on apoptosis and expression of angiogenesis factors. These results suggested that miR-575 may regulate apoptosis and angiogenesis by targeting the SOD2 gene.

In conclusion, the results of the present study suggest distinct roles of miR-575 in apoptosis and angiogenesis in villi tissue and cells. Inhibition of miR-575 may inhibit apoptosis and promote angiogenesis to prevent MA. Therefore, miR-575 may serve as a biomarker of MA, as well as a potential molecular target for the treatment of MA.

\section{References}

1. Cao W, Wenlin XU, Chen T, Wang X, Wang X, Qiu J, Chen N and Mao Y: CD4+CD25+FoxP3+ regulatory $\mathrm{T}$ cells and cytokines interact with estradiol in cases of missed abortion. Exp Ther Med 7: 417-422, 2014.

2. Sebire NJ, Thornton S, Hughes K, Snijders RJ and Nicolaides KH: The prevalence and consequences of missed abortion in twin pregnancies at 10 to 14 weeks of gestation. Br J Obstet Gynaecol 104: 847-848, 1997.

3. Zhang X, Li J, Gu Y, Zhao Y, Wang Z and Jia G: A pilot study on environmental and behavioral factors related to missed abortion. Environ Health Prev Med 16: 273-278, 2011.

4. O'Connell RM, Rao DS, Chaudhuri AA and Baltimore D: Physiological and pathological roles for microRNAs in the immune system. Nat Rev Immunol 10: 111-122, 2010.

5. Longtine MS, Chen B, Odibo AO, Zhong Y and Nelson DM: Villous trophoblast apoptosis is elevated and restricted to cytotrophoblasts in pregnancies complicated by preeclampsia, IUGR, or preeclampsia with IUGR. Placenta 5: 352-359, 2012.

6. Chatzaki E, Makrigiannakis A, Margioris AN, Kouimtzoglou E and Gravanis A: The Fas/FasL apoptotic pathway is involved in kappa-opioid-induced apoptosis of human endometrial stromal cells. Mol Hum Reprod 7: 867-874, 2001.

7. Jerzak M and Bischof P: Apoptosis in the first trimester human placenta: The role in maintaining immune privilege at the maternal-foetal interface and in the trophoblast remodelling. Eur J Obstet Gynecol Reprod Biol 100: 138-142, 2002.

8. Halperin R, Peller S, Rotschild M, Bukovsky I and Schneider D: Placental apoptosis in normal and abnormal pregnancies. Gynecol Obstet Invest 50: 84-87, 2000.

9. Chen H, Deng X, Yang Y, Shen Y, Chao L, Wen Y and Sun Y: Expression of GRIM-19 in missed abortion and possible pathogenesis. Fertil Steril 103: 138-146, 2015.

10. Nelissen EC, van Montfoort AP, Dumoulin JC and Evers JL: Epigenetics and the placenta. Hum Reprod Update 17: 397-417, 2011.

11. Miura K, Miura S, Yamasaki K, Higashijima A, Kinoshita A Yoshiura $\mathrm{K}$ and Masuzaki $\mathrm{H}$ : Identification of pregnancyassociated microRNAs in maternal plasma. Clin Chem 56: 1767-1771, 2010.

12. Hosseini M K, Gunel T, and Gumusoglu E. MicroRNA profiling in miscarriage patients. IJMM 34: S94-S94, 2014.

13. Ward RH, Modell B, Petrou M, Karagözlu F and Douratsos E: Method of sampling chorionic villi in first trimester of pregnancy under guidance of real time ultrasound. Br Med J (Clin Res Ed) 286: 1542-1544, 1983. 
14. Livak K and Schmittgen TD: Analysis of relative gene expression data using real-tie quantitative PCR and the 2(-Delta Delta C(T)) method. Methods 25: 402-408, 2001.

15. Zhu LJ, Chen YP, Chen BJ and Mei XH: Changes in reactive oxygen species, superoxide dismutase and hypoxia-inducible factor-1 $\alpha$ levels in missed abortion. Int J Clin Exp Med 7: 2179-2184, 2014

16. Savion S, Lepsky E, Orenstein H, Carp H, Shepshelovich J, Torchinsky A, Fein A and Toder V: Apoptosis in the uterus of mice with pregnancy loss. Am J Reprod Immunol 47: 118-127, 2002.

17. Choi HK, Choi BC, Lee SH, Kim JW, Cha KY and Baek KH: Expression of angiogenesis- and apoptosis-related genes in chorionic villi derived from recurrent pregnancy loss patients. Mol Reprod Dev 66: 24-31, 2003.

18. Cinar O, Kara F and Can A: Potential role of decidual apoptosis in the pathogenesis of miscarriages. Gynecol Endocrinol 28: 382-385, 2012.

19. Nair RR, Khanna A and Singh K: Association of FAS-1377 G>A and FAS-670 A>G functional polymorphisms of FAS gene of cell death pathway with recurrent early pregnancy loss risk. J Reprod Immunol 93: 114-118, 2012.

20. Levy R and Nelson D: CURRENT TOPIC: To be, or not to be, that is the question. Apoptosis In human trophoblast. Placenta 21: $1-13,2000$.

21. Kaare M, Bützow R, Ulander VM, Kaaja R, Aittomäki K and Painter JN: Study of p53 gene mutations and placental expression in recurrent miscarriage cases. Reprod Biomed Online 18: 430-435, 2009

22. Chen Y, Shen D, Gu Y, Zhong P, Xie J and Song Q: The diagnostic value of Ki-67, P53 and P63 in distinguishing partial Hydatidiform mole from hydropic abortion. Wien Klin Wochenschr 124: 184-187, 2012.

23. Taylor DD and Gercel-Taylor C: Alterations in T-cell signal transduction molecules associated with recurrent spontaneous pregnancy loss. J Reprod Immunol 63: 137-154, 2004.

24. Rajakumar A and Conrad KP: Expression, ontogeny, and regulation of hypoxia-inducible transcription factors in the human placenta. Biol Reprod 63: 559-569, 2000.

25. Lim KH, Zhou Y, Janatpour M, McMaster M, Bass K, Chun SH and Fisher SJ: Human cytotrophoblast differentiation/invasion is abnormal in pre-eclampsia. Am J Pathol 151: 1809-1818, 1997.
26. Fang Y, Yu S, Ma Y, Sun P, Ma D, Ji C and Kong B: Association of D114/notch and HIF-1a-VEGF signaling in the angiogenesis of missed abortion. PLoS One 8: e70667, 2013.

27. Arjamaa O, Nikinmaa M, Salminen A and Kaarniranta K: Regulatory role of HIF-lalpha in the pathogenesis of age-related macular degeneration (AMD). Ageing Res Rev 8: 349-358, 2009.

28. Ç̈l-Madendag I, Madendag Y, Altinkaya SÖ, Bayramoglu H and Danisman N: The role of VEGF and its receptors in the etiology of early pregnancy loss. Gynecol Endocrinol 30: 153-156, 2014.

29. Folkman J and Klagsbrun M: Angiogenic factors. Science 235: 442-447, 1987.

30. Maisonpierre PC, Suri C, Jones PF, Bartunkova S, Wiegand SJ, Radziejewski C, Compton D, McClain J, Aldrich TH, Papadopoulos N, et al: Angiopoietin-2, a natural antagonist for Tie2 that disrupts in vivo angiogenesis. Science 277: 55-60, 1997.

31. Valenzuela DM, Griffiths JA, Rojas J, Aldrich TH, Jones PF, Zhou H, McClain J, Copeland NG, Gilbert DJ, Jenkins NA, et al: Angiopoietins 3 and 4: Diverging gene counterparts in mice and humans. Proc Natl Acad Sci USA 96: 1904-1909, 1999.

32. Plaisier M, Dennert I, Rost E, Koolwijk P, van Hinsbergh VW and Helmerhorst FM: Decidual vascularization and the expression of angiogenic growth factors and proteases in first trimester spontaneous abortions. Hum Reprod 24: 185-197, 2009.

33. Seval Y, Sati L, Celik-Ozenci C, Taskin O and Demir R: The distribution of angiopoietin-1, angiopoietin-2 and their receptors tie- 1 and tie- 2 in the very early human placenta. Placenta 29: 809-815, 2008.

34. Daponte A, Deligeoroglou E, Pournaras S, Tsezou A, Garas A, Anastasiadou F, Hadjichristodoulou C and Messinis IE: Angiopoietin-1 and angiopoietin-2 as serum biomarkers for ectopic pregnancy and missed abortion: A case-control study. Clin Chim Acta 415: 145-151, 2013.

35. Xiu-Ju MA and Xiao WX: Relationship between oxidative stress and missed abortion. J Shanxi Med Univ: 2006.

36. Valdivia A, Pérez-Álvarez S, Aroca-Aguilar JD, Ikuta I and Jordán J: Superoxide dismutases: A physiopharmacological update. J Physiol Biochem 65: 195-208, 2009. 